\title{
QUAND LA COMPÉTENCE TRADUCTOLOGIQUE DÉPASSE LA COMPÉTENCE RÉDACTIONNELLE
}

Abstract. Tomaszkiewicz Teresa, Quand la compétence traductologigue dépasse la compétence rédactionnelle [How the translational competence surpasses editorial competence]. Studia Romanica Posnaniensia, Adam Mickiewicz University Press, Poznań, vol. XXX: 2003, pp. 143-157. ISBN 83232-1270-8. ISSN 0137-2475.

This article discusses a characteristic style of John Paul II's homilies, delivered during his first pilgrimage to Poland in 1979. The author touches the question of the censorship who had an influence on the way of translating these texts and ponders the limits of liberty and faithfulness of a translator who prepares his translations in a definite social and political context.

\section{PRÉLIMINAIRES}

Au début du mois de juin 2001 , la presse polonaise a rappelé un événement de l'histoire contemporaine, lourd en conséquences: la première visite du pape JeanPaul II en Pologne, en 1979, en publiant certains documents inconnus, concernant les consultations de cette visite avec les autorités de l'Union Soviétique. Pendant le pèlerinage du pape en Pologne en août 2002 de nouveau on a fait souvent l'allusion à cette première visite, en jugeant ses conséquences.

A l'époque j'ai travaillé en tant qu'interprète avec les journalistes du Figaro. Tous les textes des homélies du pape ont été disponibles, en forme écrite, ainsi que leurs traductions, dans les centres de presse, pour les besoins des journalistes. Comme ces textes ont été connus des autorités avant la visite, il était clair que le pape ne pouvait pas se permettre de dire ouvertement tout ce qu'il voulait. Or, la société polonaise, pendant sa longue histoire de lutte contre la censure, s'est habituée à comprendre entre les lignes, capter les sous-entendus, les implicites ou les non-dits, d'interpréter les allusions qui permettaient aux auteurs de «cacher» certains contenus. Pourtant cette couche délicate de significations restait souvent 
inaccessible aux récepteurs étrangers, même à ceux qui connaissaient bien le contexte socio-culturel.

Le pape partageant les mêmes expériences de la société polonaise a su introduire dans ses discours beaucoup de contenus politiques qui dépassaient la problématique religieuse.

Je peux dire que les traductions de ces textes, proposées aux journalistes, ont été très correctes du point de vue linguistique et rédactionnel, mais justement beaucoup de ces significations sous-entendues échappaient aux récepteurs étrangers, notamment aux journalistes, non-habitués à ce jeu de compréhension des implicites sous-entendus, pratiqué dans la société polonaise.

Ce n'est qu'en observant les réactions spontanées des pèlerins à certaines formulations que les joumalistes comprenaient qu'il y avait «quelque chose» làdedans. C'étaient justement ces passages que j'étais obligée de leur «expliquer», «retraduire». En analysant certains exemples, très spécifiques, du style de JeanPaul II que j'étais obligée de reformuler ou de surtraduire, j'aimerais bien réfléchir sur les limites de la fidélité et de la liberté du traducteur. En analysant l'activité traduisante de cette manière là, nous dépassons les limites de la simple compétence rédactionnelle du traducteur pour envisager les différentes dimensions de sa compétence traductologique.

\section{L'EXISTENCE DE LA CENSURE POLITIQUE EN POLOGNE}

La censure des textes pour des raisons politiques remonte en Pologne à la seconde moitié du XVII ${ }^{\mathfrak{e}}$ siècle. Tout d'abord, elle a été imposée par les autorités russes, qui ont démandé en 1650 au roi polonais Ladislas IV Vasa de punir les auteurs du livre Ladislas $I V$, roi polonais et suédois. Ces auteurs avaient décrit les victoires du roi sur les Russes à Moscou. Les autorités russes ont trouvé que ce texte était outrageant pour leur société et pour le tsar. Malgré l'indignation des intellectuels polonais, un bourreau a «exécuté» les pages inacceptables pour nos voisins, en les brûlant, d'une manière spectaculaire, sur la place du marché à Varsovie.

Cette première intervention russe, en ce qui conceme la censure des textes polonais, s'est transformée en une surveillace systématique de notre littérature. À partir de 1674, un envoyé spécial du tsar auprès de la cour polonaise, Wasyl Tjapkin, contrôlait régulièrement toute la littérature engagée en l'envoyant au Ministère des Affaires étrangères de l'époque, où une vingtaine de traducteurs la traduisaient afin d'identifier les passages qui donnaient une image négative des Russes et de confisquer les textes.

Mais les choses ne s'arrêtent pas là. À la fin du XVIII' siècle, la Pologne avait déjà été partagée entre la Prusse, la Russie et l'Autriche. Théoriquement, on n'avait 
donc pas le droit d'enseigner la langue polonaise, de publier en polonais ou de manifester son identité nationale. Face à la présence constante de la censure en Pologne, les auteurs ont adopté deux tendances. D'une part, la majorité des textes qui traitaient de la russification du peuple polonais ont été publiés ou diffusés clandestinement, sous des pseudonymes, pour éviter d'éventuelles représailles. D'autre part, les auteurs essayaient d'exprimer beaucoup de contenus de manière implicite, symbolique.

On peut dire que le savoir social sur l'obligation de soumettre tous les textes à la censure a fait naitre un code d'implicites et de sous-entendus, qui permettait aux auteurs de «cachen» certains sens derrière des formulations équivoques ou des nondits qu'un récepteur indigène savait déchiffrer et comprendre.

Après la période entre les deux guerres, la censure est revenue après 1944. Elle s'ingérait dans tous les textes littéraires, de journaux, de manuels, notamment ceux d'histoire, où certains événements historiques sont devenus des sujets tabous. Or, un grand historien polonais, Janusz Tazbir, dit, dans une récente publication (2001: 82), qu'en parlant de la censure en Pologne après la Deuxième Guerre mondiale, il ne faut pas oublier la nécessité de sa périodisation. La censure des années 19441948 n'est pas la même que celle de l'époque stalinienne, et elle a encore changé quand Edward Gierek, premier secrétaire du parti communiste, était au pouvoir dans les années 1970, époque à laquelle on a pu observer une certaine libéralisation. Mais cette libéralisation a été programmée et aussi surveillée. Finalement, il faut rappeler la période des années 1980 , qui a succédé à la première visite du pape Jean-Paul II en Pologne, en juin 1979. Cette période a débuté par la création de la Solidarité, le premier syndicat libre, et l'introduction, par la suite, de l'état de siège par le général Jaruzelski. Durant cette période-là, tout texte publié et non publié, comme par exemple la correspondance privée, portait l'inscription "censuré».

La censure opérait donc ouvertement, c'est pourquoi on savait clairement ce qu'on ne pouvait pas dire à haute voix. Il en résulte que tout le monde essayait de «lire entre les lignes» et de comprendre le vrai sens de ce qu'on publiait. Je dirais même que ce jeu de "cache-cache» faisait partie d'une convention sociale et que tout le monde cherchait des contenus sous-entendus dans toutes les publications traitant des sujets «tabous».

\section{LA THÉORIE DE LA TRADUCTION FACE AUX IMPLICITES SOUS-ENTENDUS}

Cette délicate couche de significations sous-entendues reste souvent inaccessible aux récepteurs étrangers, même à ceux qui connaissent bien le contexte socioculturel en question. Théoriquement, dans la traduction des textes censurés, on devrait introduire certaines explications supplémentaires ou supprimer les ambiguïtés pour faire comprendre aux récepteurs étrangers le vrai sens de certains 
contenus. Évidemment, les décisions concernant l'ingérence du traducteur dans l'original pour expliquer au récepteur étranger ce qui est dit de manière allusive ou implicite doivent être pondérées. Wojtasiewicz (1992:79) remarque qu'il est clair que beaucoup de ces allusions peuvent ne pas non plus être comprises par un récepteur moyen indigène, car elles sont trop érudites ou demandent une bonne connaissance du sujet. Dans ces cas-là, le rôle du traducteur ne peut pas se ramener à tout expliquer ou à tout expliciter, car le texte de la traduction serait trop appauvri.

D'après Lederer (1994: 34-35) «pour le traductologue, les présupposés de la langue (...) font partie de l'association des signifiés à la connaissance du monde; les sous-entendus sont les intentions qui fournissent l'impulsion nécessaire à la production du dire. Ils sont compréhensibles ou tout au moins supputables mais ne font pas partie du sens à transmettre en traduction».

Nous ne pouvons être d'accord avec cet avis que partiellement. Si nous acceptons l'opinion de Roberts et Pergnier (1987: 401) que «l'équivalence est fonction de choix délibérés en fonction de la nature des textes», les décisions, en ce qui concerne l'explicitation du sous-entendu, de l'inconnu, du culturel sont souvent à prendre du point de vue fonctionnel et pragmatique. Il faut savoir pour qui on traduit et quelle sera la vie de la traduction dans la société réceptrice. Quelles sont les informations ou les effets stylistiques qu'il faut transférer au récepteur étranger?

Ailleurs (Pisarska, Tomaszkiewicz 1998) nous avons proposé d'envisager l'équivalence fonctionnelle d'après trois paramètres:

- type de texte et ses fonctions;

- but de la traduction;

- réalité culturelle de la langue de départ.

Si donc un traducteur est conscient du fait qu'il vit dans une société non démocratique, où des institutions respectives soumettent des textes à la censure et veillent à ce que les publications restent opaques, mais il sait aussi que la majorité des récepteurs indigènes savent comprendre le vrai sens caché entre les lignes, il devrait introduire dans sa traduction certaines explications pour les récepteurs étrangers, inconscients de cette réalité culturelle. Evidemment, ces décisions dépendent du type de texte et du but de la traduction.

\section{DESCRIPTION DU CONTEXTE TRADUCTOLOGIQUE ENVISAGÉ}

Le corpus que je pense présenter contient des homélies de Jean-Paul II, pendant sa visite en 1979. Le Pape est resté en Pologne neuf jours, pendant lesquels il a visité neuf villes et prononcé une trentaine d'homélies au cours des messes et d'autres rencontres avec le peuple polonais ${ }^{1}$. Cette visite était gênante pour les

'En somme j'ai analysế au moins 300 pages de textes originaux en polonais ainsi que leurs traductions en français. Dans la suite je cite seulement des fragments de traductions fraçaises qu'on avait mises à la disposition des journalistes. 
autorités communistes d'au moins deux points de vue: d'une part puisque l'Église et la vie religieuse des Polonais étaient des sujets tabous, toujours censurés par les médias étatisés, d'autre part puisque ces grandes messes donnaient la possibilité aux gens de se rassembler, par milliers ou par millions, autour d'une même idée. Ouvertement, l'idée était spirituelle; implicitement, elle contestait le régime sous lequel on vivait depuis $1945^{2}$.

Évidemment, on a voulu endiguer l'enthousiasme populaire. Un jour avant la visite les journalistes français, Jean Bourdarias et Philippe Nourry s'étonnaient sur les pages du Figaro: «La presse officielle est, elle, étonnamment discrète. Pas une ligne jusqu'à présent dans ses colonnes, alors que tout le monde ici ne pense qu'à cette journée de demain».

Comme j'ai déjà dit, tous les textes des homélies du Pape ont été connus des autorités plusieurs jours avant son arrivée, et on les a traduits au Bureau de Presse du Secrétariat de l'Épiscopat de Pologne en plusieurs langues, pour le besoin des journalistes étrangers qui sont venus par milliers. Il faut dire qu'on les a traduits presque littéralement. Du point de vue linguistique, donc, on ne peut rien reprocher aux traducteurs. On peut donc dire qu'ils ont manifesté une bonne compétence rédactionnelle. Mais les textes en question contenaient justement beaucoup de contenus symboliques et implicites dont le vrai sens échappait aux récepteurs étrangers, non habitués à ce jeu de compréhension des sous-entendus, pratiqué dans la société polonaise.

Ainsi, dans beaucoup de cas, les journalistes avec qui j'ai participé à tous les événements de ces quelques jours ne pouvaient comprendre que la couche dénotative de certains passages.

Dans ce qui suit, $j$ 'aimerais bien montrer les traits caractéristiques du style de ces homélies et les opérations linguistiques qu'on devait appliquer, pendant la traduction des textes censurés pour les faire comprendre aux récepteurs étrangers. Évidemment, il s'agit d'une traduction pragmatique qui devait éclairer le sens à ceux qui, par leur métier, étaient censés diffuser le contenu de ces textes pour le besoin de leur société. Cette mission du traducteur est sûrement différente de celle d'un traducteur littéraire.

\section{MÉLANGE DES CONTENUS RELIGIEUX ET LAÏQUES}

Le premier trait caractéristique des textes analysés consiste à mélanger constamment les appels religieux et sociaux, voire politiques. Il est clair que le gouvernement a accepté la visite du Pape en tant que pèlerin qui ne pouvait se prononcer que sur la foi. Pourtant, tout en parlant de la vie spirituelle des gens et de

${ }^{2}$ Les commentaires de la presse contemporaine qui analysent la situation d'il y a plus de vingt ans disent: «Pour les autorités de l'époque, cette visite a posé un grand problème politique, entre autres puisqu'on devait consulter toutes les décisions avec Moscou» (Zaremba, 2001: 86). 
la nécessité de vivre conformément aux prescriptions de l'Église, le Pape a voulu dire aux gens ce qu'ils devaient faire pour vaincre le pouvoir oppressant. Il a voulu suggérer que dans la situation dans laquelle on vivait, être un bon Polonais signifiait être un bon chrétien. Ce raisonnement peut en choquer certains, mais cette idée faisait justement partie d'une convention sociale implicite.

On ne peut pas se permettre de laisser perdre tout ce qui est humain, polonais, chrétien sur cette terre. (Częstochowa 16.2)

L'appel de Jasna Góra n'a pas cessé d'être notre prière et notre programme. (Częstochowa 16.3)

Faites attention de ne pas vous rendre responsables des péchés des autres! (...)

Soyez sobres, veillez! (...)

Demande-toi donc, cher frère ou chère sœur, cn cette hcure dc sincérité nationale, devant la Mère et devant son cœur plein d'amour, si tu ne scandalises pas, si tu ne pousses pas au mal, si, par légèreté, tu ne charges pas ta conscience de vices et de mauvaises habitudes que les autres contractent par ta faute (...). (Częstochowa 16.2)

Dans ces trois fragments, nous voyons bien que les paroles se comprennent comme la leçon de l'Église en général, et, en même temps, nous avons tous compris qu'il y a un sens supplémentaire. Il y a tout d'abord des accents nationaux: humain, polonais et chrétien, parce qu'en Pologne, pendant les différentes périodes de l'histoire, c'est l'Église qui a aidé à préserver l'identité nationale contre les différentes formes d'ingérence étrangère. C'est pourquoi la prière à la vierge noire de Jasna Góra avait une valeur symbolique du programme politique. Les péchés dont a parlé le Pape sont évidemment les péchés normaux de tout le monde, mais aussi «les péchés des communistes» - veillez à ce que vous ne soyez pas responsables des péchés commis par les gens au pouvoir. On peut interpréter la phrase Soyez sobres et veillez comme un appel contre l'alcoolisme, mais le mot trzeźwy, traduit en français par sobre, veut dire aussi vigilant. Donc, on doit faire attention aux signes et aux mouvements provenant de l'opposition. L'heure de sincérité nationale, c'est le moment de la visite du Pape où on peut essayer de se dire certaines choses qu'on ne dirait normalement pas publiquement.

\section{DIRECTIVES POUR L'AVENIR}

La deuxième procédure du style de ces textes consistc à donner certaines directives pour l'avenir. Encore une fois on peut les lire comme les directives de la vie d'un bon chrétien, mais en même temps, il s'agissait de certaines directives politiques.

${ }^{3}$ En citant les paroles du pape Jean-Paul II, je note, entre parenthèses, la ville où l'bomélie en question a été prononcée et le numéro qu'on lui a attribué dans les traductions. Le deuxième chiffre renvoie au numéro du paragraphe du texte cité. Les originaux de tous ces textes et de leurs traductions se trouvent, entre autres, dans mes archives personnelles. 
(...) je vous prie d'accepter encore une fois tout le patrimoine spirituel qui a pour nom «Pologne» avec (wiara) la foi, la charité que le Christ a placées en nous par le saint baptême (...). (Cracovic 30.2)

Je vous prie de ne jamais perdre confiance, de ne pas vous laisser abattre, de ne pas vous décourager;

De ne pas couper vous-même les racines de notre origine.

D'avoir confiance, malgré toute votre faiblesse, et de chercher toujours la force spirituelle en Celui près duquel tant de générations de nos pères et de nos mères l'ont trouvée;

Ne vous détachez jamais de Lui;

Ne perdez jamais la liberté d'esprit, par laquelle il «rend libre» l'homme (...). (Cracovie 30.5)

Vous devez être forts de cette force qui prend sa source dans la foi? (Cracovie 30.4)

Si nous lisons bien ces fragments, qui sont d'ailleurs nombreux dans toutes les homélies, nous voyons bien que le Pape donnait des instructions aux gens en ce qui concernait leur vie spirituelle dans la foi. Évidemment, il s'agit de la croyance chrétienne, mais ce mot en polonais wiara veut dire la foi et aussi la croyance. Il s'agit donc de la foi, mais aussi, ou peut-être avant tout, de la croyance en l'avenir. Cette interprétation est possible, car le Pape a mis en quelque sorte le signe d'égalité entre le patrimoine spirituel et la Pologne. Après, il a parlé des racines de notre origine et de la liberté d'esprit. Et il a continué:

Il ne faut pas avoir peur! Il faut ouvrir les frontières! (Cracovie 30.4)

Le contexte suggère qu'il faut ouvrir les frontières pour la pénétration de l'Église, mais nous avons bien senti qu'il parlait des frontières politiques.

Et il a ajouté:

Vous devez être forts de cette force qui prend sa source dans la foi! Vous devez être forts de la force de la foi! Vous devez être fidèles! Aujourd'hui plus qu'à aucune autre époque, vous avez besoin de cette force. Vous devez être forts de la force de l'espérance qui conduit à la parfaite joie de vivre et ne permet pas de contrister l'Esprit Saint. (Cracovie 30.4)

Il parlait donc de l'espérance et de la force dont on avait besoin aujourd'hui plus qu'à aucune autre époque. Il a voulu dire que le temps est venu pour faire quelque chose, pour se mettre contre le régime, en cherchant de l'aide dans l'Église.

Et finalement, il a exprimé le vou que:

(...) la prière pour la victoire de l'ordre moral dans ce moment difficile de notre histoire (...) soit le principal moteur de tous les travaux, de toutes les tâches et tous les programmes qui sont et seront entrepris en terre polonaise. (Cracovie 30.3)

Il n'a évidemment pas pensé seulement au programme de l'Église, mais aussi aux autres programmes possibles, entrepris par les différentes forces de l'opposition. Avant sa rencontre avec les jeunes, à Cracovie, on n'a distribué aucun texte. Ce n'était pas une messe, donc aucune homélie n'était prévue, seulement une prière ensemble. Mais, c'est à Cracovie que le Saint-Père a dit aux jeunes: «Prenez 
cette croix et plantez-la partout où vous vivez, où vous travaillez, où vous allez à l'école (...)». Je dois dire que cette directive a été concrétisée pendant de longues années suite à l'état de siège. Pour manifester ou protester contre le pouvoir, les gens allumaient des bougies, rangées dans des endroits importants des villes, en forme de croix gigantesques. C'était des manifestations silencieuses contre lesquelles il était très difficile de lutter.

En face de ces doubles significations du contenu, significations comprises comme la leçon de l'Église, mais aussi comme un programme politique, la compétence traductologique aurait dû se manifester par une surtraduction, une certaine explicitation des sous-entendus. Evidemment officiellement ce n'était pas possible, car les textes des traductions ont été, eux aussi, censurés.

\section{EXPLOITATION DE L'AMBIGUÏTÉ}

Cette procédure que nous venons de décrire, à savoir le mélange des appels à caractère religieux et des propositions d'une certaine lutte contre les forces au pouvoir, a été possible grâce à l'exploitation du phénomène de l'ambiguîté.

\subsection{DOUBLE SENS EN POLONAIS QUI N'EXISTENT PAS EN FRANÇAIS}

Le premier double sens, que j'ai déjà évoqué, c'est le mot clé de plusieurs homélies wiara, qui veut dire la foi, mais aussi la croyance, non seulement en Dieu, mais en l'avenir, en les programmes, en d'autres valeurs. Ce terme a été exploité à plusieurs reprises par le Pape avec son double sens.

Le deuxième double sens qui apparaît aussi à plusieurs reprises, mais qui a été surtout important dans l'Homélie de la messe au sanctuaire martial de Jasna Góra, c'est niewola. D'une part, ce mot veut dire la servitude à la Mère de Dieu Reine du royaume de Pologne, faisant allusion à un acte du roi Jean-Casimir, au XVII siècle, qui a proclamé la Sainte Marie reine de la Pologne. D'autre part, ce mot veut dire esclavage et cette signification se rapporte aux Polonais en tant qu'esclaves du régime, des forces étrangères, de la situation politique, etc. L'utilisation de cette deuxième signification est encore plus claire quand elle est comparée au champ sémantique du mot wolność (liberté). Le Pape parlait donc de la «liberté de l'Église dans le monde et en Pologne et son droit d'annoncer le message de salut!» Dans cette formulation, il y a encore un double sens du mot salut. De la version française, nous comprenons qu'il s'agit du "salut de l'âme», mais en polonais on a dit zbawcza misja qu'on peut comprendre comme «mission de sauver, libéren». Le Pape a donc suggéré que l'Église en Pologne a aussi, entre autres, la mission de «libérer les gens de l'esclavage». Et il a appelé cela l'neuvre de la liberté divine, qui a été traduit en français par l'auvre de l'auteur divin, où le terme liberté, tellement lourd de significations, disparaît. 


\subsection{IMPLICITES FONDÉS SOCIALEMENT (STÉRÉOTYPES SOCIAUX)}

À côté des termes plurisémantiques, il y en a d'autres qui ont des contenus implicites, que l'on peut découvrir grâce à ce que Kerbrat-Orecchioni appelle: la "compétence encyclopédique partagée» (1986: 163), qu'on appelle aussi le «bagage cognitif» (Lederer 1994: 37). Il s'agit des termes enracinés dans la société en question, qui ont une certaine valeur affective stéréotypée. R. Amossy (1997) souligne que ces jugements stéréotypés expriment symboliquement l'identification des gens à une collectivité. Dans notre cas, il s'agit de certains termes se rapportant aux droits de l'homme, à sa dignité, à sa liberté, à son patriotisme qui jouissent d'une valeur particulière dans la société polonaise. Je ne veux pas dire que ces termes existent seulement dans notre société, mais chez nous, ils sont particulièrement importants.

Ainsi, le pape Jean-Paul II a inauguré sa visite en Pologne par la messe à Varsovie. Le sujet principal de son homélie était le millénaire du christianisme en Pologne, mais ce texte est plein de contenus implicites. Il a dit ouvertement: «On ne peut pas comprendre l'histoire de la Pologne (...) sans Jésus Christ.(...) On ne peut pas comprendre, sans Jésus, cette nation dont le passé extraordinaire a été aussi très difficile». (Varsovie 5.3b)

Et il a continué en disant: «Jésus Christ nous apprend la dignité de l'hornme et ses droits ainsi que la dignité et les droits de la Nation».

Un peu plus loin il a dit: «Aujourd'hui je prie avec vous pour que le Christ ne cesse pas d'être pour nous l'enseignement pour l'avenins. Et tout de suite il a comparé Jésus Christ au soldat inconnu (dont le tombeau se trouve à côté de l'endroit où il a célébré la messe) en disant que ce soldat a péri sur les champs de bataille du monde entier pour prouver qu'il n'y a pas de justice en Europe sans une Pologne indépendante.

Tous ces termes, comme dignité de l'homme, droits de l'homme, avenir, justice, Pologne indépendante, évoquaient à l'époque plusieurs sens dans l'esprit des récepteurs indigènes, toute une série de connotations, liées au passé et à la situation présente.

Un autre terme ayant plusieurs sous-entendus, c'est le mot prison. Il a été utilisé par le Pape dans quelques homélies, notamment à Varsovie, près du tombeau du soldat inconnu, et à Auschwitz, dans l'ancien camp de concentration. Théoriquement, il a évoqué les différentes périodes de l'histoire où les Polonais ont été des prisonniers politiques, mais tout le monde a compris qu'il parlait aussi de l'époque du régime stalinien ainsi que de toute la période communiste.

Ici la tradition n'est pas une limitation: c'est un trésor, c'est une richesse spirituelle, c'est un grand bien commun, qui se confirme en tout choix, en tout acte noble, en toute vie authentiquement vécue en chrétien. (Cracovie 30.2)

Ces mots du Pape nous donnent l'impression qu'il s'agit effectivement de la vie en chrétien, mais tout de suite il faut comprendre que cette vie en chrétien, c'est 
justement le modèle auquel s'opposait le régime communiste, pour lequel plusieurs ont été emprisonnés et pour lequel beaucoup de gens ont perdu la vie.

L'explication des sous-entendus dus à l'ambiguîté sémantique de certains termes est plus facile du point de vue de la compétence traductologique que l'explication des implicites fondés socialement. Ceux derniers véhiculent souvent une certaine charge affective, manifestée par une société, mais inconnue ou incompréhensible par une autre. L'interprétation traductologique, dans ce cas là, peut devnir trop subjective.

\section{L'INTERTEXTUALITÉ}

Le procédé suivant, qui permet d'obtenir l'effet de sous-entendu, consiste à exploiter l'intertextualité. Il s'agit de faire référence aux textes connus de la société pour déclencher certains sentiments ou certaines attitudes affectives. Ce procédé peut se réaliser de deux manières différentes.

\subsection{RÉFÉRENCE AUX TEXTES INDIGÈNES}

On peut se référer aux textes enracinés dans la culture indigène et méconnus des étrangers. Ces références sont nombreuses dans les homélies du pape Jean-Paul II, c'est pourquoi nous ne pouvons pas les citer toutes. Mais anrêtons-nous sur les trois exemples les plus significatifs.

- Le premier hymne de la Pologne s'appelle Bogurodzica, qu'on a traduit en français par Mère de Dieu. Cette traduction ne véhicule pas tout le sens historique du terme. Cet hymne a toujours été chanté par les chevaliers, et plus tard par les soldats, avant les grandes batailles. On attache une grande importance à sa signification symbolique, qu'on ne pouvait pas rendre par la simple traduction Mère de Dieu. Le Saint-Père a cité ce titre archaïque en parlant de la Sainte Marie, tout en faisant référence à sa signification symbolique: hymne national chanté avant les grandes batailles pour se donner du courage.

- Le deuxième exemple concerne la littérature polonaise romantique. Historiquement, le romantisme embrasse la période du grand partage, où la Pologne, était partagée entre la Prusse, la Russie et l'Autriche. Toute la littérature de cette époque des grands romantiques polonais, tels que Mickiewicz, Słowacki et Krasiński, représente le héros romantique qui consacre sa vie à la patrie, la Pologne, symbolisée par le Christ crucifié. De là provient l'expression Pologne: Christ crucifié des Nations. Le Pape a souvent fait allusion à cette image, qui, à vrai dire, reste peu compréhensible pour le récepteur étranger sans une explication supplémentaire. 
- Le troisième exemple conceme ce qu'on appelle la «langue de bois» des mass médias polonais de l'époque communiste. Ce langage, plein d'expressions toutes faites, essayait de montrer la supériorité du système communiste sur les régimes occidentaux. Il reposait sur certains axiomes et expressions toutes faites qui revenaient sans cesse dans les disours des hommes politiques et dans la presse. Le pape Jean-Paul II a souvent fait référence à ces opinions pour les mettre en doute ou pour les réfuter. C'est très clair, par exemple, dans son homélie à Nowa Huta, ville ouvrière près de Cracovie, quand il a dit:

La nouvelle croix est apparue quand, sur les territoires des anciennes campagnes des alentours de Cracovie devenu territoire de Nowa Huta, sont venus des hommes nouveaux pour commencer un nouveau travail. (Nowa Huta 27.2)

Il a dit cela parce que les autorités avaient propagé l'idée que le monde du travail n'avait pas besoin de l'Église et il a continué:

On ne peut pas séparer la croix du travail humain. On ne peut pas séparer le Christ du travail humain. (...) Le christianisme et l'figlise n'ont pas peur du monde du travail. $11 \mathrm{n}$ 'ont pas peur du système fondé sur le travail. Le Pape n'a pas peur des travailleurs. (Nowa Huta 27.2)

Par ces paroles, il a effectivement fait allusion aux opinions propagées par le pouvoir que le régime communiste est un régime de la classe ouvrière qui n'a pas besoin de la tradition chrétienne. Il l'a souligné encore une fois:

Mais rappelez-vous cette unique chose: le Christ n'approuvera jamais que l'homme soit considéré - ni qu'il se considère lui-même - seulement comme un instrument de production, et qu'il soit apprécié, estimé et évalué selon un tel critère. (Nowa Huta, 27.2)

Nous voyons clairement la polémique avec l'idéologie marxiste-léniniste, obligatoire à l'époque dans notre pays. Dans une traduction explicative on aurait pu, par exemple ajouter un petit élément: Le Pape n'a pas peur des travailleurs, comme le prétendent les autorités communistes. Mais, évidemment ce n'était pas possible à cause de la censure.

\subsection{RÉFÉRENCE AUX TEXTES RELIGIEUX}

Mais l'intertextualité peut fonctionner différemment. Le pape Jean-Paul II a introduit dans ses discours beaucoup de citations de l'Évangile et des Écrits Saints que tous les chrétiens connaissent et comprennent dans le cadre religieux. Or, en citant ces textes, on peut vouloir les interpréter d'une manière dépassant la simple leçon de l’Église.

La cathédrale de Varsovie (...) a été presque complètement détruite pendant l'insurrection. Celle dans laquelic nous nous trouvons est un édifice totalement nouveau. C'est aussi un signe de vie nouvelle, polonaise et catholique, qui trouve son centre dans la cathédrale. 
C'est le signe de ce que le Christ a dit autrefois «Détruisez ce temple, et en trois jours je le rebâtirai» (Jn,2,19). (Varsovie 3.4)

En se servant d'un fragment connu, le Pape a fait allusion à deux sujets tabous. Tout d'abord, il a évoqué l'insurrection de Varsovie en août 1944, quand l'Armée rouge, stationnant de l'autre côté de la Vistule, a permis aux Allemands de détruire complètement Varsovie. Ensuite, il a fait allusion au fait que les communistes, en venant au pouvoir, ont voulu détruire des églises, ou les transformer en dépôts et qu'ils ont oppressé les croyants.

De plus, il a continué en se référant aux événements historiques polonais remontant au $\mathrm{XI}^{e}$ siècle, soit le conflit entre le roi Boleslas le Téméraire et l'évêque de Cracovie, Stanislas. Ce conflit s'est terminé par la condamnation à mort de Stanislas par le roi. Cet événement symbolise le conflit entre le pouvoir et l'Église. Le Pape a aussi fait allusion à cette histoire:

(...) Stanislas a dit de lui-même au roi Boleslas «Détruis cette Église et le Christ, au long des générations, ta reconstruira».(Varsovie 3.4)

C'est comme s'il voulait faire référence à la situation en Pologne communiste où nous avons assisté à la lutte constante entre le pouvoir et l'Église, cette dernière signifiant l'opposition.

\section{ALLUSIONS AUX ÉVÉNEMENTS HISTORIQUES}

Ce demier exemple nous montre encore un domaine qui nécessite certains éclaircissements pour le récepteur étranger, à savoir la référence à certains événements de l'histoire d'une nation qui ont des valeurs symboliques ou qui, pendant certaines périodes, sont devenus tabous. Nous avons déjà évoqué le problème de l'interpétation historique de l'insurrection de Varsovie en 1944 et de la symbolique de l'évêque Stanislas «qui est appelé patron de l'ordre moral» (Częstochowa 14.4).

Parmi les sujets tabous évoqués par Jean-Paul II, il y a, par exemple, l'allusion faite à la christianisation des peuples slaves. Il a énuméré les Croates, les Slovènes, les Bulgares, les Moraves, les Slovaques, les Tchèques, etc., et à un certain moment, il a dit «ll faut qu'on rappelle le baptême de la Russie à Kijev en 988» (Gniezno 8.5), qu'on n'a pas traduit en français. C'est peut-être un petit élément, mais c'est aussi la manifestation de la censure qui est intervenue pour supprimer ce qui appartenait à ces sujets tabous.

Un autre sujet délicat de différents points de vue, ce sont les territoires à l'ouest et au nord de la Pologne. Dans la traduction, on lit:

Le pape [Paul VI] qui s'est tant dépensé pour normaliser la vie de l'Église en Pologne, particulièrement en ce qui concerne l'organisation actuelle des terres de l'ouest et du nord (...). (Częstochowa 10.2) 
Dans le texte polonais, le Pape a dit: «(...) en Pologne, particulièrement en ce qui concerne ses terres actuelles occidentales et nordiques».

La différence n'est pas grande, mais quand même l'expression figée Ziemie Zachodnie i Pólnocne déclenche toute une série de connotations qui rappellent en même temps les territoires polonais à l'est qu'on a intégrés à l'Union Soviétique, tandis que les gens qui y habitaient ont été transportés à l'ouest et au nord.

\section{0. ÉLÉMENTS AJOUTÉS}

Pour compléter cette image des difficultés qui surgissent devaut un traducteur qui a affaire aux textes truffés d'implicites et de sous-entendus, il faut ajouter qu'il y avait certains passages, pas très nombreux, mais souvent significatifs, que le Pape a ajouté pendant les homélies et qui ne se trouvaient pas dans les textes distribués aux joumalistes. Par exemple, les autorités ont tout fait pour contrôler la participation des citoyens aux messes. On avait prévu des cartes d'invitation qu'on a distribuées dans les paroisses. Et surtout, on n'a pas voulu que la rumeur concernant cette visite parvienne aux autres pays communistes. Or, certains pèlerins des pays voisins sont parvenus, à l'aide des curés polonais, à venir et à participer aux événements.

Pendant la messe à Cracovie, le Pape les a salués en les nommant frères des Carpates, et il a exprimé le souhait que d'autres peuples slaves, qui sont nos frères, puissent être en ce moment avec nous.

À un autre endroit, il a exprimé le vœu que le gouvernement populaire permette à l'Église de parler ouvertement de Jésus Christ.

\section{D'AUTRES MANIFESTATIONS DE LA CENSURE DANS LES TRADUCTIONS}

Finalement, la censure dans les traductions est visible par le fait que, dans les originaux, certains fragments les plus significatifs sont soulignés pour attirer l'attention des récepteurs sur ce qui est le plus important. Dans les traductions, souvent, ces fragments ne sont pas soulignés, ou pour donner l'impression de l'identité des deux versions, on a souligné d'autres passages.

\section{EN GUISE DE CONCLUSION}

Nous savons bien qu'une multiplicité de facteurs interviennent dans le décodage des unités de contenu et que plusieurs compétences des récepteurs entrent en jeu. Kerbrat-Orecchioni le dit de la manière suivante: 
Étant donné la multiplicité des facteurs intriqués dans cet écheveau fort complexe que constitue la compétence interprélative globale, il n'est pas étonnant que la quête du sens d'un énoncé quelconque soit toujours plus ou moins tâtonnante, et son résultat toujours plus ou moins aléatoire (Kerbrat-Orecchioni, 1986: 301).

Il n'empêche que dans des conditions déterminées, un facteur important pour comprendre les implicites ou non-dits sont des compétences cognitjves partagées par les membres de la même société. Dans ces cas-là, la traduction doit être, comme le dit Fuchs,

une paraphrasc. c'est-à-dire un déplacement sémantique, qui procède par des réćquilibrages à la fois par surdétermination et par sous-détermination du sens: le texte d'arrivéc dit tantôt «plus» que le texte d'origine (notamment en explicitant certains implicites), et tantôt «moins» (Fuchs, 1996: 86).

Il aurait donc été souhaitable, dans notre exemple, de tenter une surdétermination, c'est-à-dire d'expliciter tous les sous-entendus contenus dans les homélies du pape Jean-Paul II, prononcées pendant sa visite historique en Pologne. Or, comme nous l'avons vu, ces textes avaient été censurés de sorte que beaucoup de messages n'ont pu être transmis que de manière implicite ou sous-entendue. Le sens était possible à déchiffrer par ceux qui partageaient la même compétence sociale et culturelle. Les autorités ont veillé à ce qu'on ne pratique aucune surtraduction, c'est-à-dire à ce qu'on n'explicite guère, ce qui pouvait nuire à une bonne réception de ces messages.

Personne ne pouvait reprocher à ces traductions qu'elles aient été mal faites du point de vue de la correction linguistique, mais elles ne remplissaient pas complètement la vocation d'une bonne traduction, qui devrait, dans la mesure du possible, donner accès au sens au récepteur étranger. Ce n'est que grâce à la compréhension de leur mission par certains traducteurs, en tant que médiateurs entre les cultures, que beaucoup de journalistes ont pu interpréter correctement les messages du pape Jean-Paul II en 1979.

Et même si maintenent, après tous les changements qui sont survenus en Europe centrale et orientale, nous pouvons penser qu'il s'agissait de la moindre des choses, à l'époque, le rôle des traducteurs et interprètes dans la transmission des informations était indéniable. Mais il faut le dire ouvertement, il s'agissait de contacts face à face et non pas de traductions «officielles», car celles-ci devaient correspondre aux exigences du pouvoir.

Or, ce contexte traductologique spécifique nous incite à reprendre la discussion sur les limites de la fidélité et de la liberté du traducteur. La fidélité, notion fondamentale de chaque théorie traductologique, reste, à mon sens, une notion mal définie. Il est clair que chaque tentative d'interprétation du vouloir dire de l'auteur est, en quelque sorte, subjective. Pourtant, est-il possible de se libérer de sa propre compréhension? A qui doit-on rester fidèle? A l'auteur? A celui qui commande la traduction? A soi-même? A sa conscience? A sa morale? En posant ces questions 
nous dépassons les limites de la simple compétence rédactionnelle du traducteur pour envisager les différentes dimensions de sa compétence traductologique.

Pourtant, les réponses à ces questions restent souvent ouvertes, car, comme nous l'avons démontré, le travail du traducteur est souvent conditionné par les différents paramètres situationnels. Ainsi, le contexte traductologique peux, dans certains cas, influencer la quilité de la traduction.

\section{OUVRAGES CITÉS}

A mossy, R., Herschberg Picrrot, A. (1997), Stéréotypes et clichés. Paris: Nathan Université.

Berman, A. (1985), La traduction comme épreuve de l'étranger, Texte, 4, p. 67-81.

Bourdarias, J., Nourry, Ph. (1979), Ia Pologne reçoit demain Jean.Paul Il. Comment endiguer l'enthousiasme populaire?, Le Figaro, 1.06.1979, p. 9.

Fuchs, C. (1996), Les ambigü̈tés du françaris. Paris: Ophrys.

Lederer, M. (1994), La traduction aujourd'hui. Modèle interprétatif. Paris: Hachctte.

Kerbrat-Orecchioni, C. (1986), L'implicite. Paris: Armand Colin.

Pisarska, A., Tomaszkiewicz, T. (1998), W'spólczesne tendencje przekladoznawcze. Poznań: Wydawnictwo Naukowe UAM.

Roberts Roda, P., Pergnicr, M. (1987), L'équivalence en traduction. Mcta, vol. 32, nº 4, p. 392 402.

Tazbir, J. (200I), Slowa na stosie. Dhugie dzieje cenzury w Polsce, Polityka 9 (2287), p. 80-82.

Wojtasiewicz, O. (1992), Wstęp do teorii thumaczenia. Warszawa: TEPIS.

Zaremba, M. (2001), Utrzymać Watykan na linii, Polityka 21 (2299), p. 86-89. 